\title{
An Intelligent System for Document Retrieval in Distributed Office Environments *
}

\author{
Uttam Mukhopadhyay, $†$ Larry M. Stephens, Michael N. Huhns, $\ddagger$ and \\ Ronald D. Bonnell \\ Center for Machine Intelligence, University of South Carolina, \\ Columbia, SC 29208
}

\begin{abstract}
MINDS (Multiple Intelligent Node Document Servers) is a distributed system of knowledge-based query engines for efficiently retrieving multimedia documents in an office environment of distributed workstations. By learning document distribution patterns, as well as user interests and preferences during system usage, it customizes document retrievals for each user. A two-layer learning system has been implemented for MINDS. The knowledge base used by the query engine is learned at the lower level with the help of heuristics for assigning credit and recommending adjustments; these heuristics are incrementally refined at the upper level.
\end{abstract}

\section{Introduction}

Documents are used in computerized office environments to store a variety of information. This information is often difficult to utilize, especially in large offices with distributed workstations, because users do not have perfect knowledge of the documents in the system or of the organization for their storage. The goal of the MINDS project is to develop a distributed system of intelligent servers that (1) learn dynamically about document storage patterns throughout the system, and (2) learn interests and preferences of users so that searches are efficient and produce relevant documents $[1,2]$. The strategy adopted for evaluating a set of learning heuristics that are applicable to this goal is presented. In particular, this paper describes the heuristic evaluation testbed, distance measures for metaknowledge, document migration heuristics, evidence assimilation techniques, and results of a system simulation.

*This research was supported in part by NCR Corporation.

†New address: Computer Science Department, General Motors Research Laboratories, Warren, MI 48090.

₹New address: Artificial Intelligence Department, Microelectronics and Computer Technology Corporation, 9430 Research Boulevard, Austin, TX 78759

Received June 17, 1985; accepted August 30, 1985.

(c) 1986 by John Wiley \& Sons, Inc.

\section{Distributed Workstation Environment}

\section{A. Organization of Documents}

Queries regarding documents are frequently based on the contents of the documents. Automatic text-understanding systems could conceivably process these queries by reading the documents, but would be expensive to develop and use. The names of documents provide clues to their contents, but names are not descriptive enough for reliable processing of content-based queries. However, a set of keywords may be used to describe document contents: the retrieval of documents can then be predicated on these keywords as well as on other document attributes, such as author, creation date, and location. Complex qualifiers, which are conjunctions or disjunctions of predicates on these attributes, may also be used. Each document is thus represented by a surrogate containing its attributes. The document and its surrogate are subsequently updated or deleted as dictated by system usage. Surrogates occupy only a fraction of the storage space required by the documents, but usually contain enough information for users to determine whether a document is useful.

The presumed office environment consists of a network of single-user workstations. Each user may query the system about his own locally-stored documents or about those stored at other workstations. These documents are not permanently located but may migrate to other workstations. Multiple copies of documents are allowed, but documents stored at one location must have unique names.

\section{B. The User's Perspective}

In typical distributed document management systems, document directories are either centralized or distributed, with or without redundancy [3]. However, the directory 
information is consistent throughout the system; information is stored redundantly only to reduce directory access time. The algorithm for document retrieval consists of matching predicates for retrieval with the document properties stored in the directory. The documents for which the match is successful are then retrieved from the indicated storage addresses. Since the directory information is consistent throughout the system, the response to a query is the same without regard to the identity of the query originator.

In a large system, the response to a user's query may consist of many documents, only a few of which may be relevant to that user. Also, the set of documents relevant to a second user may be quite different from that to the first, even though their queries are identical. The problem appears to originate from a lack of specificity in formulating the query. A judicious choice of predicates would apparently cause all the documents that are irrelevant to the query originator to be rejected. However, this would require a sophisticated query language, rich enough to allow the expression of a user's short-term and long-term goals, plans, and interests. A comprehensive framework for document surrogates would also be required. Formulating queries would be extremely cumbersome and the increased power of the system would be offset by the additional effort demanded from the user.

In the absence of any information about the user, whether explicitly stated in the query or embedded in the system knowledge base, a response will necessarily consist of a superset of the sets of relevant documents described by the query from the perspective of each user. Usertransparency in a large multiple-user environment may thus cause a query response to contain a large number of irrelevant documents. It is our view that systems of the future need to maintain models of their users in a background mode in order to make document searches more efficient and productive without burdening the user.

MINDS is a distributed document server with some special characteristics that allow personalized document retrieval. Additional information, in the form of personalized document metaknowledge, is stored at each workstation to allow the system to scan the document bases of all system users in a best-first fashion from the viewpoint of the query originator. MINDS maintains (at each workstation) models of both the current system state and the local user's document preferences.

\section{Query Processing}

\section{A. Workstation Interactions}

The MINDS system shares tasks, knowledge, and metaknowledge for cooperation among the workstations. A complex query is processed by first decomposing it into simpler subtasks, with the help of locally-stored metaknowledge, such that the search space for a subtask is limited to the documents owned by one user. Subtasks are then transmitted to the respective workstations where they are processed. Responses to the subqueries are transmitted back to the workstation that initiated the subqueries, where the results are synthesized and ranked in decreasing order of relevance as estimated by the metaknowledge. If the subquery is content-based, relevant metaknowledge is also sent to the query originator along with the documents and surrogates constituting the response to the subquery. The transmitted metaknowledge may be used for updating the metaknowledge of the receiver in accordance with the learning strategy. Other activities, such as creating, deleting, and copying documents, require cooperation among the workstations. Again, metaknowledge may be modified as a side-effect of these activities.

\section{B. Metaknowledge}

The relevance of a keyword to a particular document is assumed to be either zero or one, in accordance with traditional (Boolean) indexing. Further, query-term weights are also assumed to be either zero or one. (See Bartschi [4] or Kraft and Buell [5] for a survey of fuzzy and generalized information retrieval techniques.) These assumptions are made to simplify the query processing at each workstation so that the distributed aspects of our research would be emphasized: metaknowledge is used for finding the best locations for the query processing to take place. Each metaknowledge element (Fig. 1) is a four-tuple with fields for two users, a keyword, and a certainty factor in the closed interval $[0,1]$. The metaknowledge element, (Smith, Jones, compiler, 0.8), represents the following:

Smith's past experience suggests that the possibility of finding relevant documents on compilers among those owned by Jones is high ( 8 on a scale of 10$)$.

Formally, given $U$, the set of all users, and $K$, the set of all keywords, the metaknowledge function, $M$, is the mapping

$$
M: U \times U \times K \rightarrow[0,1] .
$$

The metaknowledge is partitioned among the workstations such that if $U i$ is the subset of users at workstation $i$, then only the metaknowledge for $U i \times U \times K$ is stored at that workstation.

A certainty factor [6] provides a basis for ordering the search for documents. It reflects (1) the breadth of information at a workstation pertaining to a specific keyword, (2) how useful documents concerning this keyword have proven to be in the past, and (3) how recently the workstation has acquired its information. The metaknowledge is first initialized with default values of certainty factors. A set of heuristic learning rules defines the constraints for modifying these values during system usage.

If a user had metaknowledge for each document rather than for each user of the system, the knowledge would be precise. However, the disadvantages of this approach are (1) for an average of $n$ documents per user, the meta- 


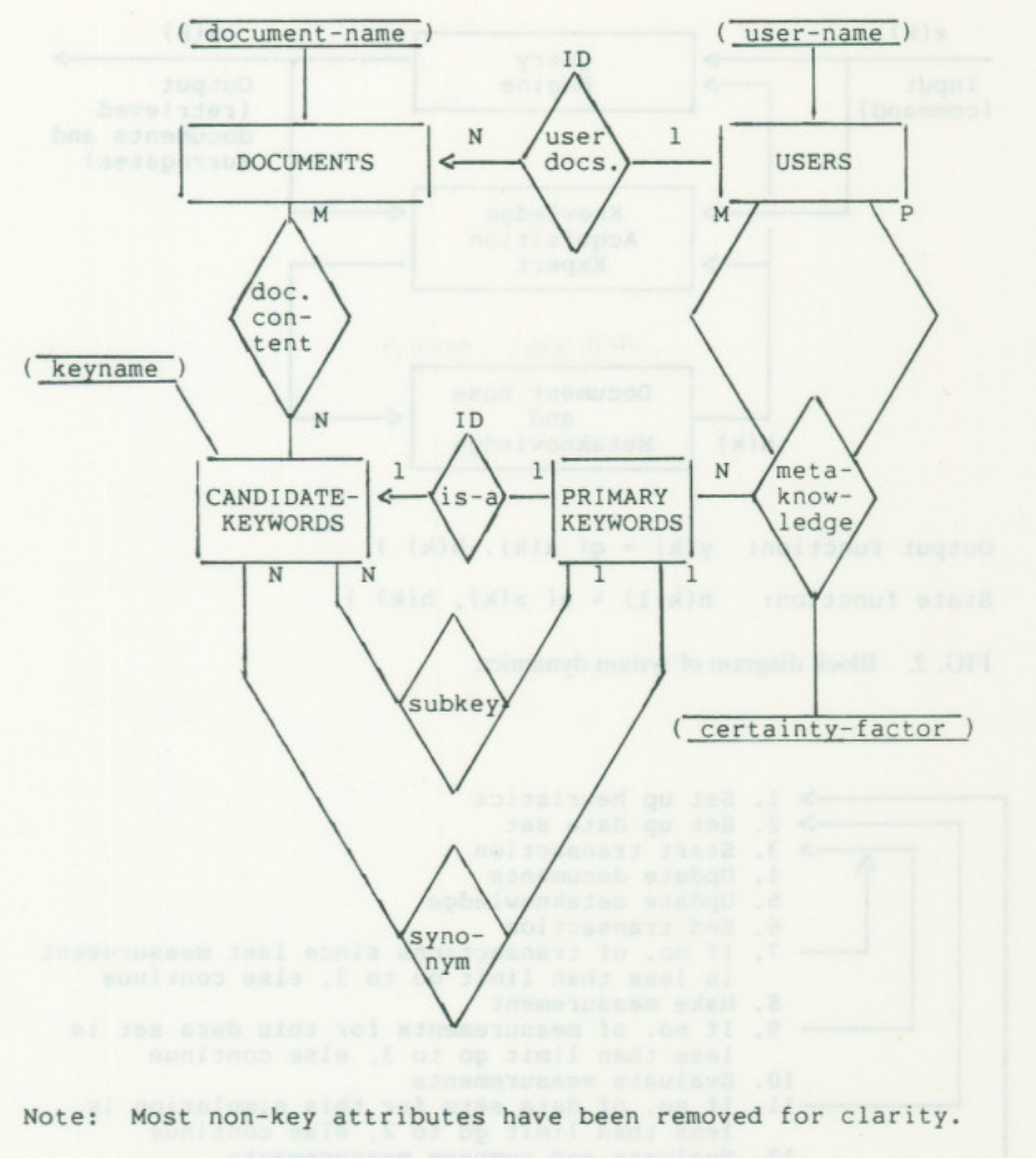

FIG. 1. Entity-relationship diagram for the metaknowledge of the system.

knowledge overhead would be $n$ times as much, and (2) for new documents, no prediction of relevance can be made. On the other hand, there are positive correlations among the documents owned by a particular user, so that conceptual-level properties may be assigned to correlated clusters of these documents. Future additions to a cluster would have properties similar to the cluster prototype. For example, the metaknowledge stored by Smith associating the document base of Jones with the keyword compiler is a conceptual-level property of Jones' document base. This property can be exploited by Smith to facilitate document searches and is therefore stored at Smith's workstation.

\section{The Learning Testbed}

\section{A. The Learning Cycle}

MINDS is being developed for operation in a wide range of office environments. The state of an environment at any instant of time is given by the content and configuration of the metaknowledge and document bases of the system. Commands issued by users comprise the system inputs, and retrieved documents and surrogates constitute the outputs. The state of the system changes as a result of executing a command [7]. These system dynamics are shown in Figure 2.
A testbed has been implemented to develop a robust body of learning heuristics for MINDS. A strategy for this development, outlined in Figure 3, is based on simulating the performance of different versions of MINDS, each implemented with a different set of learning heuristics. Each simulation is run on a representation of a specific office environment, consisting of an initial database of documents and their locations, the initial metaknowledge of the users, and a command sequence representing plausible document transactions. The performance of the set of heuristics is measured periodically during the simulation. This set is then tested on other simulated environments. Based on the evaluations of each simulation, heuristics are discarded, added, or modified; the simulations are then repeated. Good heuristic refinement rules (metaheuristics) expedite the search for an optimal set.

\section{B. Domain Modeling and Knowledge Representation}

The practical value of the heuristics developed in the testbed depends on the validity of the office models used in the simulations. An office is modeled by aggregate descriptors such as the number of users and the relative frequencies of certain commands. These descriptors are used to generate a distributed document base, metaknowledge for each user, and a command sequence. 


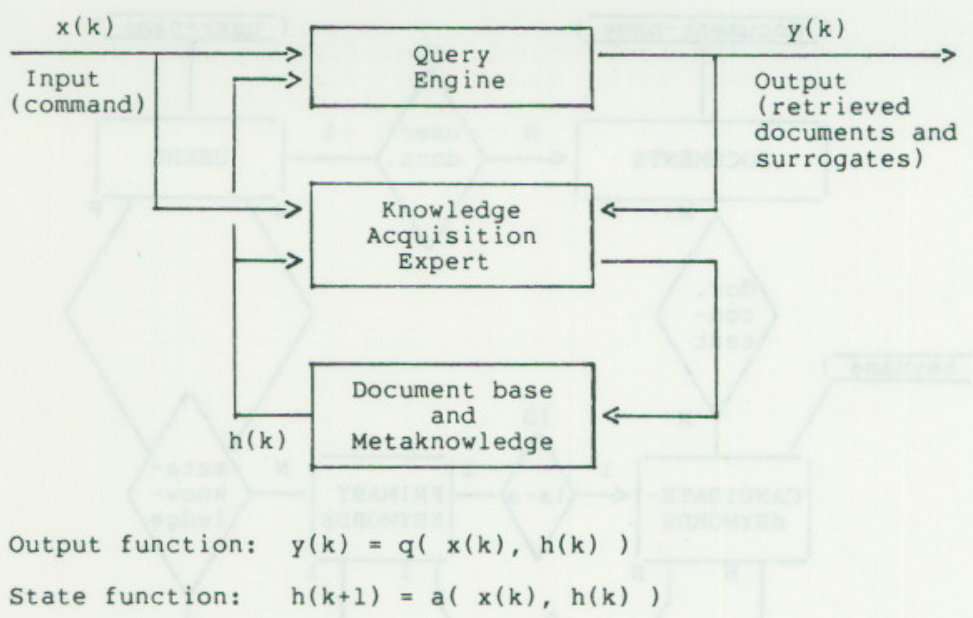

FIG. 2. Block diagram of system dynamics.

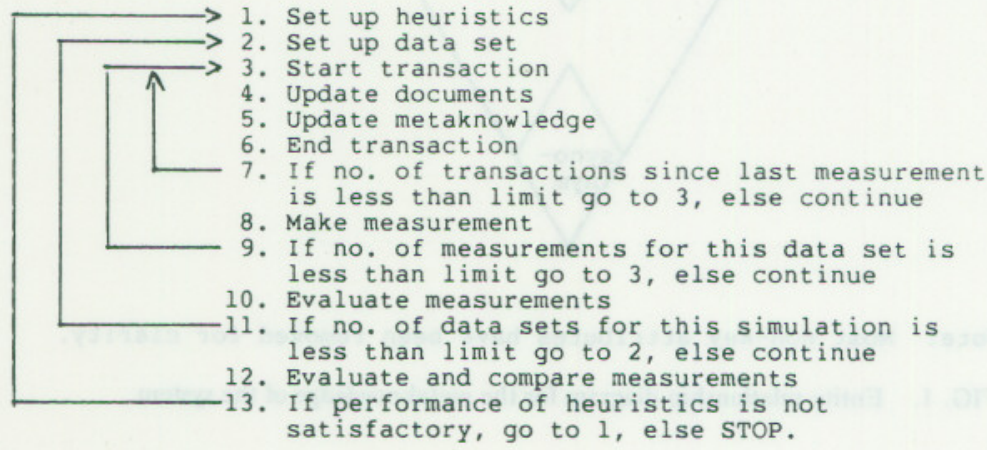

FIG. 3. The heuristics refinement cycle.

Although document retrievals may be based on several types of predicates such as authorship and location, only content-based (keyword) retrievals are considered in the simulations since other types of retrievals do not modify the metaknowledge. Commands which do not affect the system state, and thus are not important for learning, are also not simulated.

Each document is given a name and several descriptive keywords. A READ operation with a keyword-based predicate is a retrieval that culminates in the reading of one or more documents from the set returned. In an actual system, a user would then provide a relevance factor on a $[0,1]$ scale for each document read. If the documents were to be retrieved and read by another user, the relevance factors would probably be different. Also, if the same user evaluated the same documents in terms of some other keyword, the relevance factors would probably be different. In the simulations, the contents of each document are not stored, only the relevance factor it would be accorded by each user in terms of each descriptive keyword.

If documents are distributed uniformly such that there is no preferred sequence of workstations to search, the metaknowledge will not prove helpful. However, instead of employing an exhaustive search strategy, people in offices (computerized or otherwise) always seem to rely on past experiences to order their searches in a best-first fashion. This suggests that the distribution of knowledge in offices is not uniform.

The correlation among the documents owned by a particular user is modeled in the testbed by biasing the relevance factors associated with the documents. For example, if Jones' documents on compilers are biased from Smith's viewpoint by 0.2 , then the relevance factors associating Smith with compilers in documents owned by Jones will have a uniform distribution between 0.2 and 1.0. A bias of -0.4 would cause a distribution between 0 and 0.6 . The bias is mutual in that:

$$
\begin{aligned}
& \text { BIAS (Smith, Jones, compiler) = } \\
& \text { BIAS (Jones, Smith, compiler). }
\end{aligned}
$$

A typical document base is shown in Figure 4.

Metaknowledge is stored as shown in the example of Figure 5. Each user has metaknowledge which captures his personal view of the dispersion of relevant documents and consists of certainty factors for all combinations of 


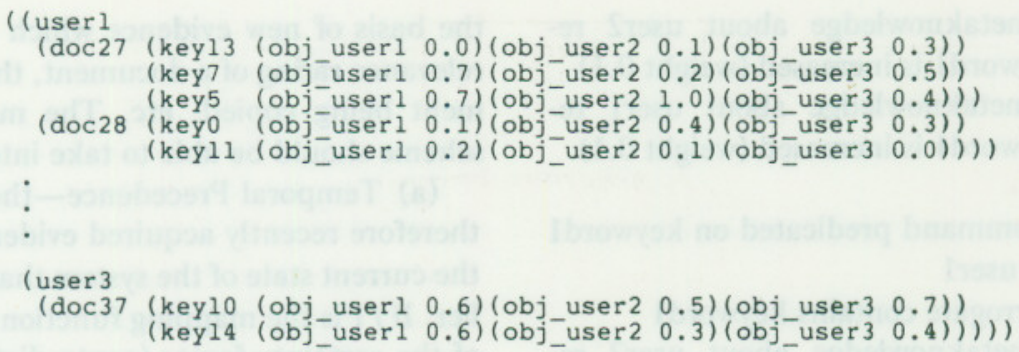

FIG. 4. Document base representation in the testbed.

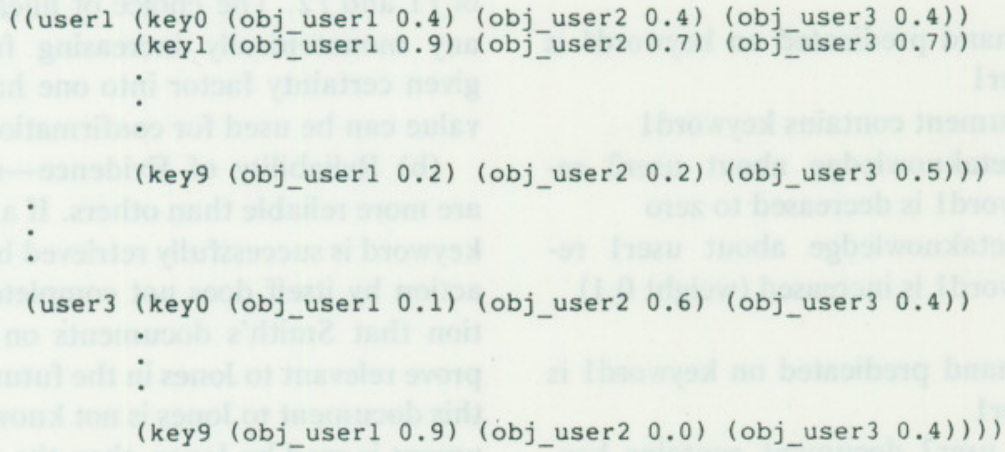

FIG. 5. Metaknowledge representation in the testbed.

users and keywords, including his view of his own documents. A system of $n$ users and $m$ keywords would result in $n$ times $m$ certainty factors in each of the $n$ metaknowledge sets.

Two choices were considered for initializing the metaknowledge at the start of the experiment. The first, an unbiased assignment of certainty factors (say 0.5 ), would result in ties for determining the best locations to search; if the conflict-resolution strategy is to choose the first location to appear in the list, then the system would tend to learn about users placed at the top of the list earlier than those placed near the end. The second initialization strategy would be to randomly allocate certainty factors, possibly with a uniform distribution, to ensure that the learning progresses in an unbiased fashion. The second strategy was adopted for the simulations reported in this paper.

\section{Metaknowledge Update Heuristics}

The heuristics for updating metaknowledge are based on the paradigm of an intelligent office-worker who conducts an ordered search for documents based on past experiences in the office environment. When Smith asks Jones for documents on compilers and Jones provides one or more documents that are relevant to him, Smith learns that Jones' document base is likely to continue having useful documents on compilers. If Jones has no documents on compilers, or if none of the documents on compilers are relevant to him, Smith will learn that this may not be a good place to search for documents on compilers in the future. In either case, Jones may assume that Smith will continue searching other locations and acquire documents on compilers from some other user. Consequently, Jones will increase his belief in Smith's ability to provide documents on compilers in the future. This increase in belief will be modest since Smith's newly acquired knowledge on compilers may not be of the type relevant to Jones.

(1) Initial Set of Heuristics. Move and copy commands do not explicitly appear in the command sequence for this set of simulations. They do appear, however, in some of the learning heuristics for document migration. The results described in Section 4.D were generated by the following set of heuristics:

Heuristic 1. (also applicable for the DELETE part of MOVE)

IF a document is deleted

THEN no metaknowledge is changed

Heuristic 2.

IF a document is created by user1

THEN metaknowledge of user1 about user1 regarding each keyword of the document is increased to 1.0 (the maximum relevance).

Heuristic 3.

IF a retrieve command predicated on keyword1 is issued by user1

AND at least one user2 surrogate contains keyword1 
THEN (a) user1 metaknowledge about user2 regarding keyword1 is increased (weight 0.1 )

(b) user2 metaknowledge about user1 re-

Heuristic 4. garding keyword1 is increased (weight 0.1 )

IF a retrieve command predicated on keyword1 is issued by user1

AND no user2 surrogate contains keyword1

THEN (a) user1 metaknowledge about user2 regarding keyword1 is decreased to zero

(b) user2 metaknowledge about user1 re-

Heuristic 5. garding keyword1 is increased (weight 0.1 )

IF a read command predicated on keyword1 is issued by user1

AND no user2 document contains keyword1

THEN (a) user1 metaknowledge about user2 regarding keyword1 is decreased to zero

(b) user2 metaknowledge about user1 regarding keyword 1 is increased (weight 0.1 )

Heuristic 6.

IF a read command predicated on keyword1 is issued by user1

AND at least one user2 document contains keyword 1

THEN (a) user1 metaknowledge about user2 regarding keyword1 is changed, based on the highest relevance of all user2 documents regarding keyword1.

(b) user2 metaknowledge about user1 regarding keyword1 is increased (weight 0.1 )

Heuristic 7.

IF a read command predicated on keyword1 is issued by user1

AND document1 owned by user 2 contains keyword1

AND the relevance of document1 to user1 by way of keyword1 exceeds the move-copy threshold

AND user1 does not have document1

THEN (a) user1 copies document1 from user2

(b) metaknowledge of user1 about user1 regarding keyword1 of the document is increased to 1.0

Heuristic 8 .

IF a read command predicated on keyword1 is issued by user1

AND user1 has copied document1 from user2

AND the maximum relevance of document1 to user 2 by way of any keyword is less than the delete threshold

THEN document1 is deleted from the document base of user2

These heuristics have also been written in first-order logic and as OPS5 rules for implementation purposes.

(2) Assimilation of Evidence. The learning heuristics shown above enable the metaknowledge to be changed on the basis of new evidence which typically consists of the relevance rating of a document, the observation of a document being copied, etc. The metaknowledge updating scheme should be able to take into account

(a) Temporal Precedence-the system is dynamic and therefore recently acquired evidence is more indicative of the current state of the system than evidence acquired earlier. If $f 1$ is the mapping function for a downward revision of the certainty factor (contradiction) and $f 2$ is the mapping function for an upward revision (confirmation), then $f 2(f 1(x)) \geq f 1(f 2(x))$, for all $0 \leq x \leq 1$. This is illustrated in the bottom graph of Figure 6 for linear functions of $f 1$ and $f 2$. The choice of linear functions is arbitrary; any monotonically increasing function which maps a given certainty factor into one having a greater (smaller) value can be used for confirmation (contradiction).

(b) Reliability of Evidence-some types of evidence are more reliable than others. If a surrogate with a desired keyword is successfully retrieved by Jones from Smith, this action by itself does not completely support the proposition that Smith's documents on compilers are going to prove relevant to Jones in the future, since the relevance of this document to Jones is not known. However, if this document is read by Jones, then the relevance value assigned by him constitutes reliable evidence. The reliability of the source is also important for evaluating the metaknowledge sent by a user. When Smith offers metaknowledge to Jones about documents on compilers, Jones will pay heed to it only if he has found Smith to be a reliable source of documents on compilers in the past.

(c) Degree of Support-the degree of support for a proposition may vary. When a user is asked if the document he has read is relevant to him, his answer does not have to be limited to "yes" or "no," but may be a value in the range $[0,1]$.

(d) Saturation Characteristics-when the initial certainty factor for a metaknowledge element is high, additional confirmatory evidence will not change (increase) it substantially. However, if the evidence were to be contradictory, the change (decrease) in confidence factor would be large. The situation is exactly reversed when the initial certainty factor is low.

The metaknowledge updating scheme presented here has all these features and is based on two linear functions that map the current certainty factor to a new one (Fig. 6). The choice of linear functions is arbitrary as discussed previously. The first function deals with confirmatory evidence that causes upward revision while the second one deals with contradictory evidence that causes downward revision. When a surrogate with a keyword is retrieved successfully, the revised certainty factor is given by $(1-r)$ $* f 1(x)+r * f 2(x)$, where $f 1$ and $f 2$ are the functions dealing with downward and upward revision, $x$ is the original certainty factor, and $r$ is the reliability of this type of information (typically 0.1 ). For example, if the evidence supports a proposition to a degree of $r=0.7$, the mapping function is the weighted average of the two original functions (see Fig. 7). 
Conf irmation

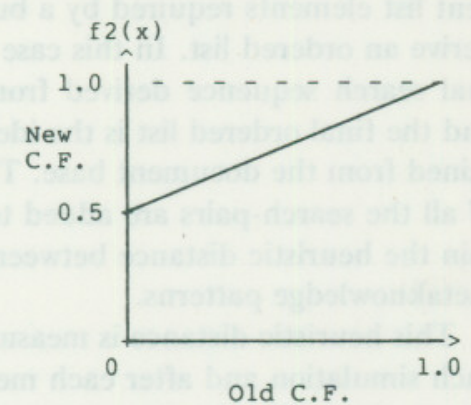

Contradiction

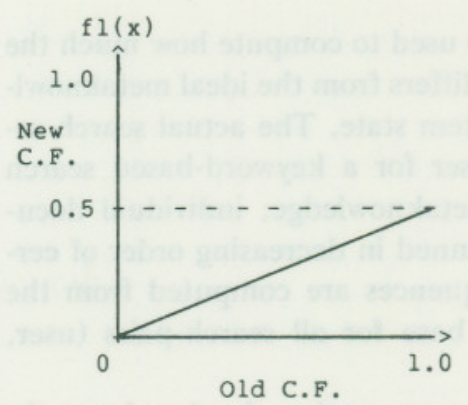

Precedence Characteristics

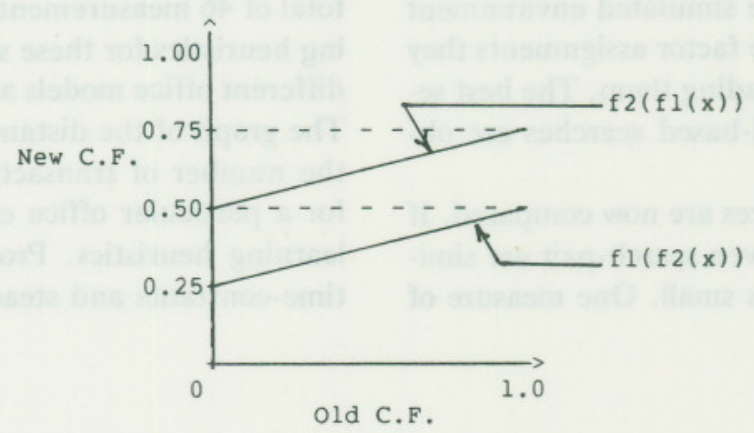

FIG. 6. Update functions for metaknowledge certainty factor and temporal precedence characteristics.
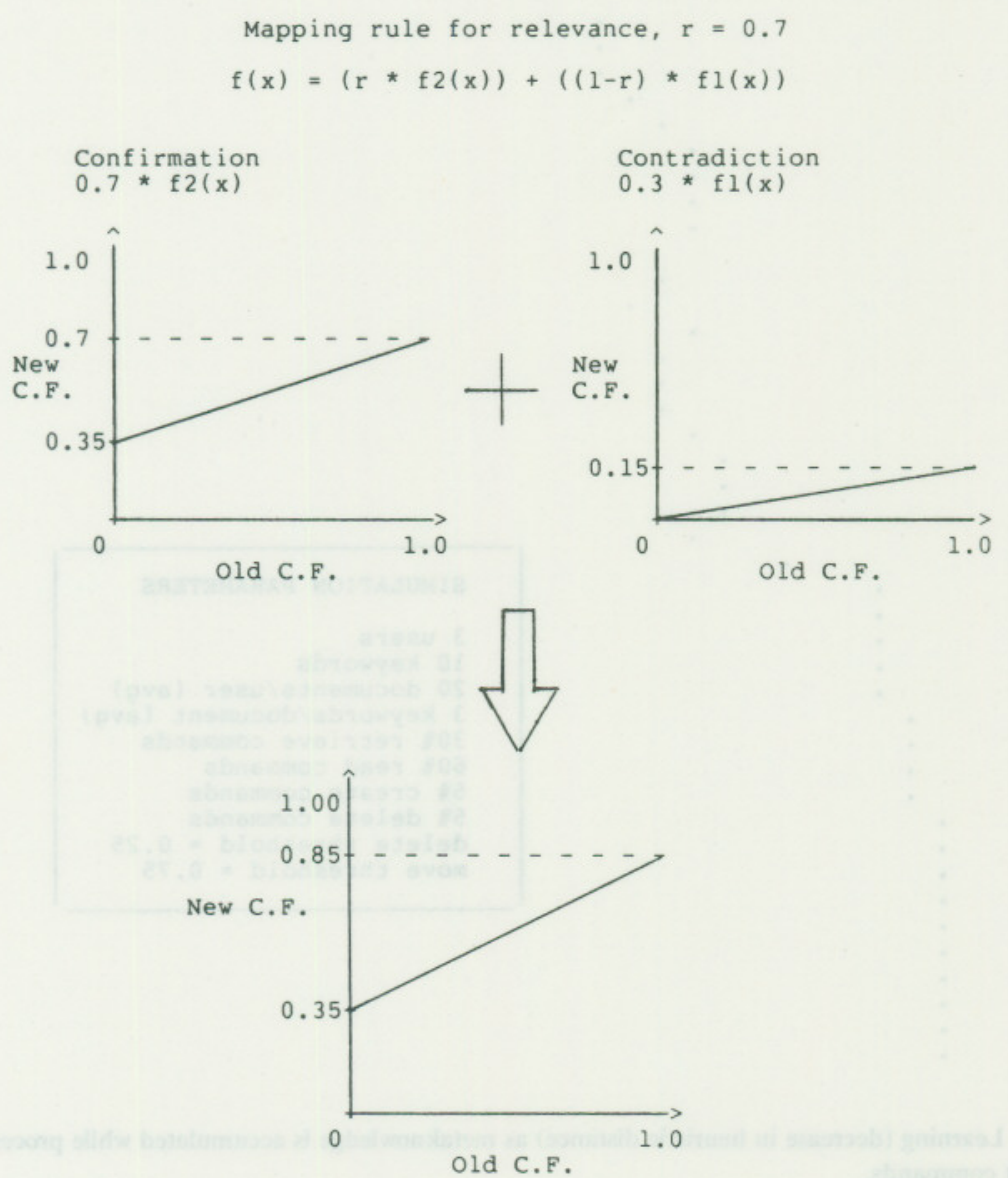

FIG. 7. Updating scheme for metaknowledge certainty factors. 


\section{Learning by Decreasing the Heuristic Distance}

A heuristic function is used to compute how much the current metaknowledge differs from the ideal metaknowledge for a particular system state. The actual search sequence adopted by a user for a keyword-based search would depend on his metaknowledge; individual document bases would be scanned in decreasing order of certainty factors. These sequences are computed from the current metaknowledge base for all search-pairs (user, keyword).

The ideal search sequences, on the other hand, are obtained from the current document base. For reasons explained earlier, documents in the simulated environment are augmented with the relevance factor assignments they would have elicited from users reading them. The best sequences for conducting keyword-based searches are obtained from this information.

The two sets of search sequences are now compared. If the two search sequences for a given search-pair are similar, the distance between them is small. One measure of disorder in a list is the number of exchanges between adjacent list elements required by a bubble-sort procedure to derive an ordered list. In this case the initial list is an actual search sequence derived from the metaknowledge, and the final ordered list is the ideal search sequence obtained from the document base. The measure of disorder of all the search-pairs are added together in order to obtain the heuristic distance between the current and ideal metaknowledge patterns.

This heuristic distance is measured at the beginning of each simulation and after each measurement cycle of ten transactions. For the simulation results shown in Figures 8-11, 450 transactions (commands) were executed and a total of 46 measurements were made. Although the learning heuristics for these simulations were kept unchanged, different office models and usage patterns were employed. The graph of the distance measurements as a function of the number of transactions produces the learning curve for a particular office environment and particular set of learning heuristics. Properties of these graphs, such as time-constants and steady-state values, are being used to

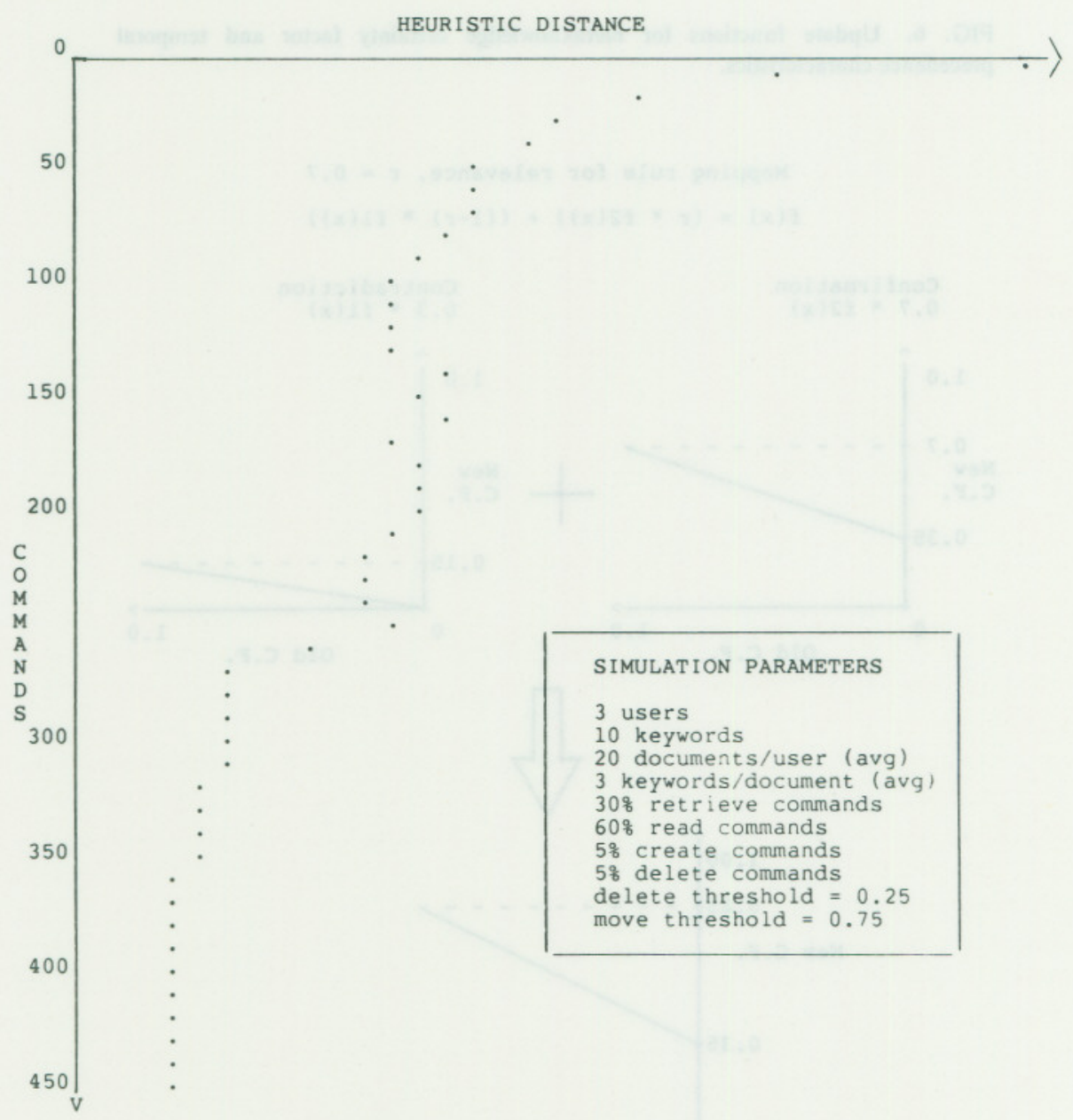

FIG. 8. Learning (decrease in heuristic distance) as metaknowledge is accumulated while processing document commands. 


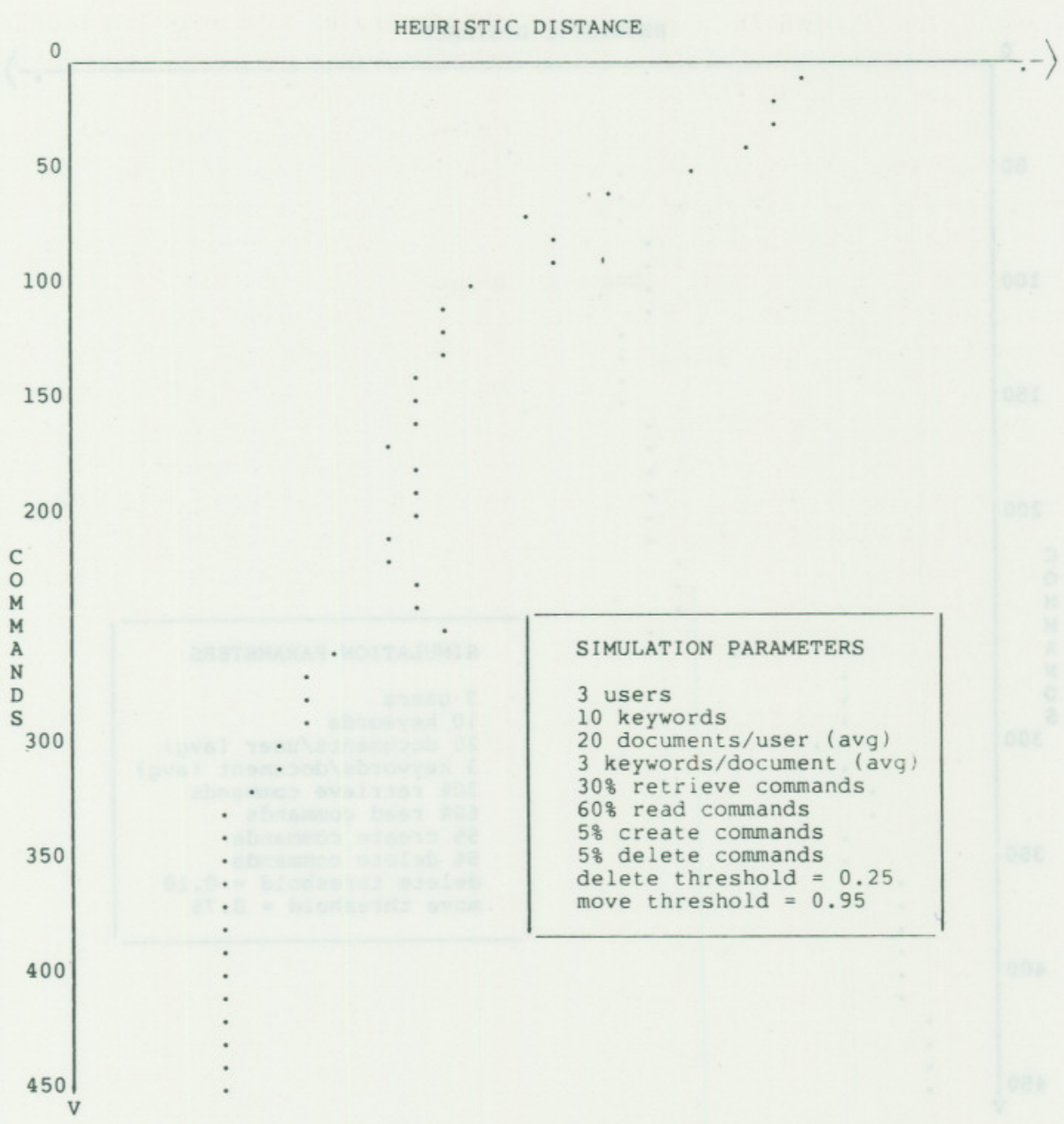

FIG. 9. Learning (decrease in heuristic distance) as metaknowledge is accumulated while processing document commands.

evaluate the performance of the heuristics in order to derive metaheuristics.

\section{The Multilayered Learning System Model}

Buchanan et al. [8] have proposed a general model for a learning system (LS) based on four functional components, a performance element, a learning element, a critic, and an instance selector. Each component has bidirectional communication links to a blackboard containing the knowledge base as well as all temporary information used by the learning system components.

The performance element is responsible for generating an output in response to each new stimulus. The instance selector draws suitable training instances from the environment and presents them to the performance element. The critic evaluates the output of the performance element in terms of a performance metric and suggests adjustments to the learning element, which makes appropriate changes to the knowledge base. The LS operates within the constraints of a world model which is the conceptual framework of the system, containing assumptions and methods for domain activity and domain-specific heuristics [9].

The world model can not be modified by the LS that uses it, but it may be altered by a higher-level system based on the observed performance of the LS. This system may itself be a learning system. Incremental refinement of the world model can thus be accomplished in a higherlevel learning system (LS2) whose performance element is the learning system at the lower level (LS1). Several wellknown LSs have been characterized using this multilevel framework $[8,10]$.

Dietterich et al. [11] have developed a simple model for learning systems that incorporates feedback from the performance element to the learning element. The included knowledge base is not specified as a blackboard. This model has been used to examine the factors that affect the design of the learning element.

The MINDS two-level testbed for heuristic refinement has been mapped into an integrated framework, combin- 


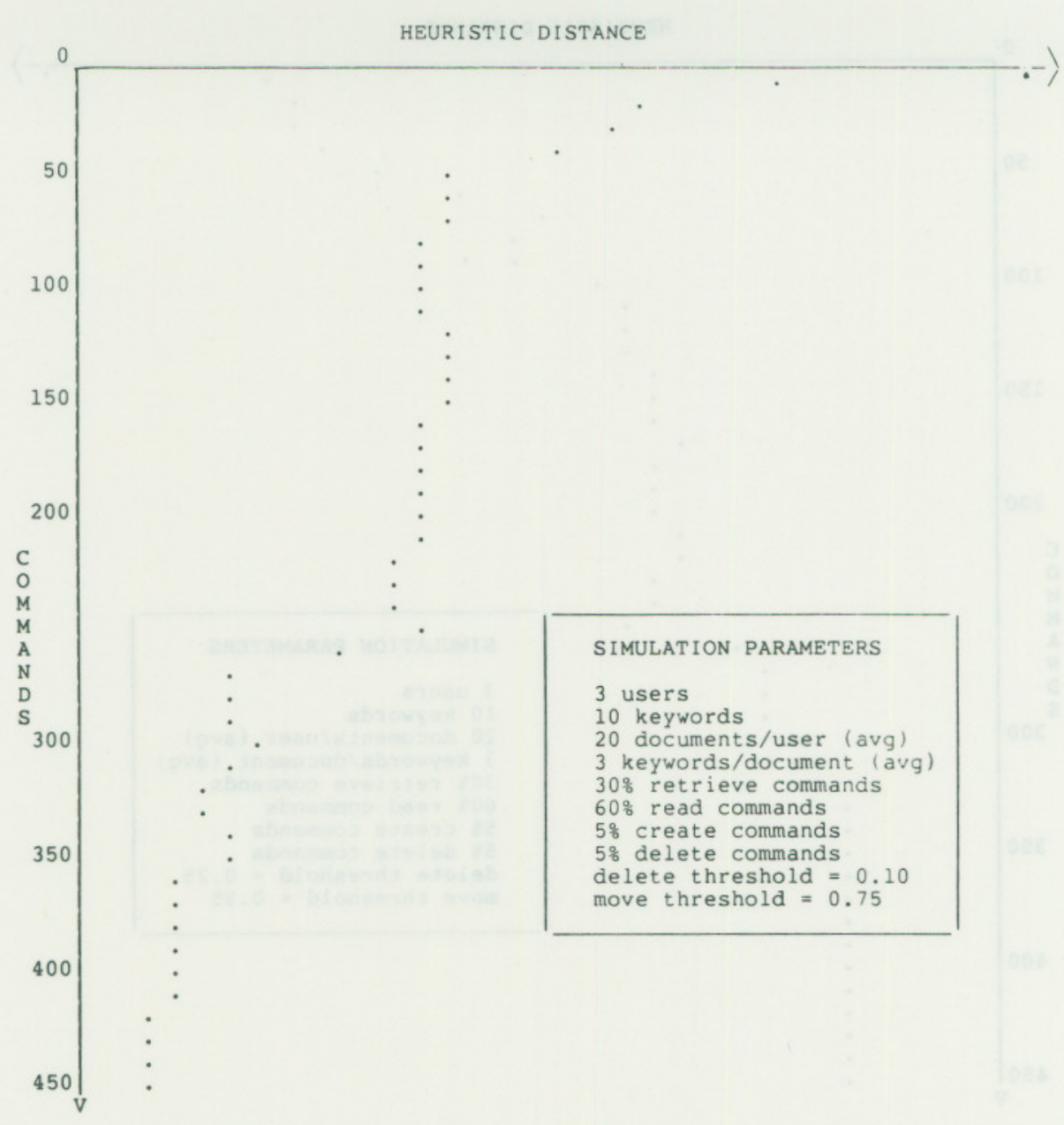

FIG. 10. Learning (decrease in heuristic distance) as metaknowledge is accumulated while processing document commands.

ing features of both of the above general models. This framework is shown below:

\section{The User Layer}

Goal: Learn to customize document searches for individual users in a dynamic setting.

\section{The Upper Layer (LS2, Fig. 12)}

Purpose: Improve the performance of LS1 by selecting a good set of learning heuristics.

Environment: All command sequences, initial metaknowledge configurations and initial document distributions that comprise the training set.

Instance Selector: Chooses an interesting environment (a combination of command sequence, metaknowledge configuration, and document distribution) with help from the critic.

Critic: Evaluation. Uses metaheuristics (presently sup- plied by the authors) to analyze learning curves for LS1 with the current set of learning heuristics.

Diagnosis. Singles out heuristics that are not useful.

Therapy. Selects new heuristics to replace useless ones or suggests modifications for existing ones. Also suggests interesting environments for testing a new set of heuristics.

Learning Element: Redefines the current set of heuristics as recommended by the critic.

World Model: The LS1 world model along with the set of metaheuristics for updating the LS1 heuristics, the method for evaluating the performance of LS1, and the scheme for heuristic updating.

\section{The Lower Layer (LS1, Fig. 13)}

Purpose: For each user learn a good set of confidence factors to predict the outcomes of all keyword-based searches of individually-owned document bases.

Environment: Set of all possible combinations of document distributions and user commands. 


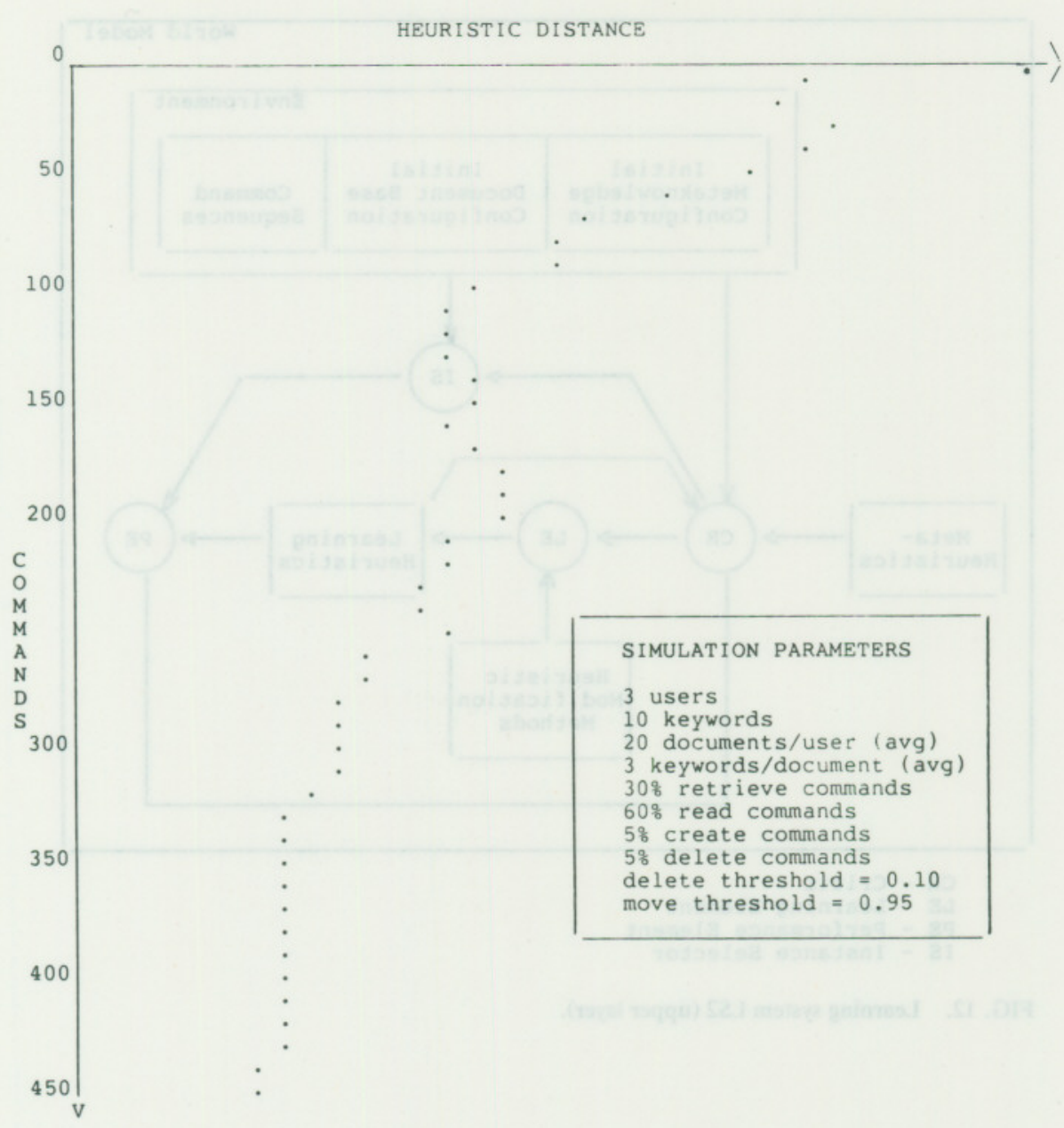

FIG. 11. Learning (decrease in heuristic distance) as metaknowledge is accumulated while processing document commands.

Performance Element: Uses the document metaknowledge (set of confidence factors) to plan a document base search sequence for a user. Also executes other commands which may change the document distribution pattern.

Instance Selector: The next command is read from a predefined sequence of commands. The document distribution pattern chosen to be part of the environment for the execution of a command is simply the document configuration arrived at after execution of the last command.

Critic: Evaluation. Examines the result of searching a target document base with the help of the learning heuristics.

Diagnosis. Determines that the document bases be searched in the order that would have proved most fruitful as determined from the results.

Therapy. Recommends increases and/or decreases of certainty factors.

Learning Element: Adjusts the certainty factors in the metaknowledge according to the critic's recommendation.

World Model: Representations of the document base, knowledge base and commands, the metaknowledge updating scheme, and the learning heuristics for the evaluation of results and recommendation of therapies.

\section{Conclusions and Plans}

The testbed has provided a useful tool for developing the heuristics needed for learning the document storage patterns of individuals using distributed workstations. Environments of this type are modeled by higher-level descriptions which are used to generate a document base, metaknowledge, and a plausible transaction sequence for each user.

Simulations are run for these environments and the resulting learning curves are used to identify a better set of heuristics. A search strategy is required for rapid convergence to an optimal set of heuristics for a given office environment. Part of the current research effort is to identify metaheuristics that would help refine existing heuristics, as well as discover new ones. 

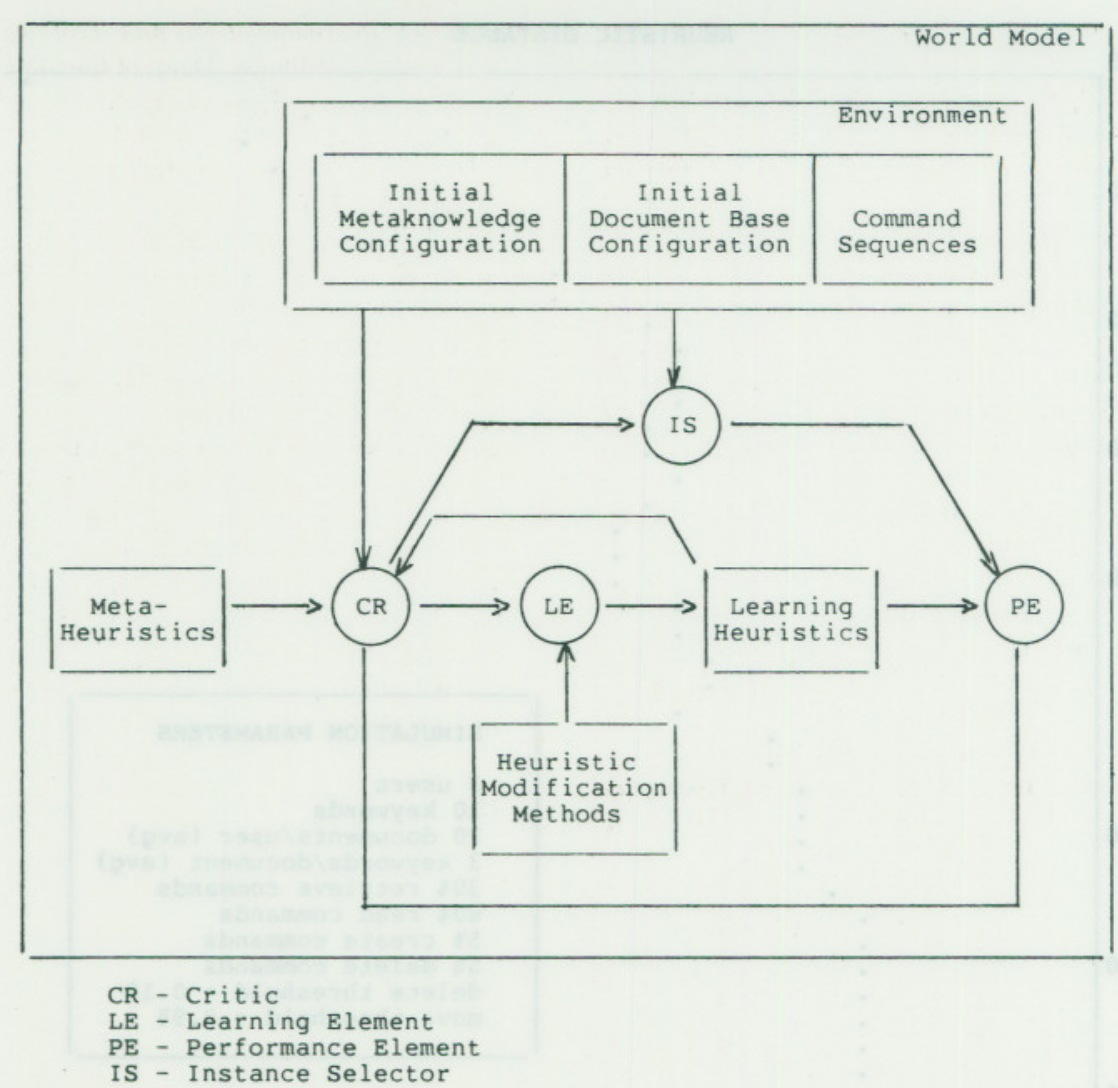

FIG. 12. Learning system LS2 (upper layer).

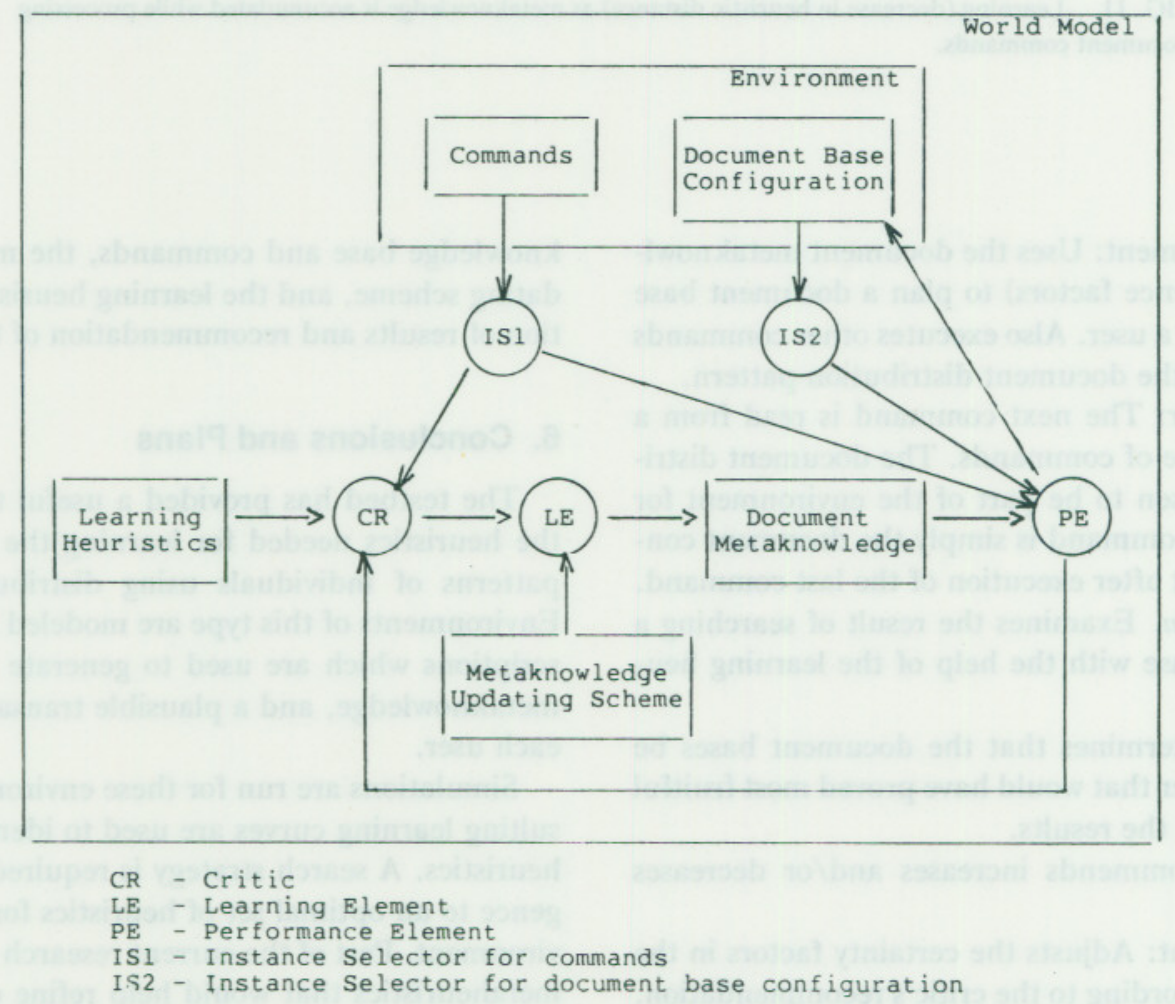

FIG. 13. Learning system LS1 (lower layer). 


\section{References}

1. Bonnell, R. D.; Huhns, M. N.; Stephens, L. M.; Mukhopadhyay, U. "MINDS: Multiple Intelligent Node Document Servers," Proceedings IEEE First International Conference on Office Automation, December 1984, 125-136.

2. Bonnell, R. D.; Huhns, M. N.; Stephens, L. M.; Mukhopadhyay, U. "A Distributed Expert System Approach to the Intelligent Filing System," USCMI Technical Report 84-17. Columbia, SC: University of South Carolina; November 1984.

3. Rothnie, J. B.; Goodman, N. "A Survey of Research and Development in Distributed Database Management," Proceedings IEEE Third International Conference on Very Large Data Bases. 1977, $30-44$.

4. Bartschi, M. "An Overview of Information Retrieval Subjects." IEEE Computer. 18(5):67-84; 1985.

5. Kraft, D. H.; Buell, D. A. "Fuzzy Sets and Generalized Boolean Retrieval Systems." Int. J. Man-Machine Studies. 19:45-56; 1983.

6. Buchanan, B. G.; Shortliffe, E. H. "Reasoning Under Uncertainty," Rule-Based Expert Systems. Reading, MA: AddisonWesley; 1984.

7. Lenat, D. B.; Hayes-Roth, F.; Klahr, P. "Cognitive Economy In a Fluid Task Environment,” In: R. S. Michalski, Ed., Proceedings of the International Machine Learning Workshop. Urbana, IL: University of Illinois, Dept. of Computer Science; 1983.

8. Buchanan, B.; Mitchell, T.; Smith, R.; Johnson, Jr., C. "Models of Learning Systems," In: J. Belzer et al., Eds., Encyclopedia of Computer Science and Technology, Vol. 11. New York: Marcel Dekker; 1977:24-51.

9. Winston, P. H. "Learning Structural Descriptions from Examples," The Psychology of Computer Vision. New York: McGrawHill; 1975:157-210.

10. Rendell, L. A. "Conceptual Knowledge Acquisition in Search," In: L. Bolc, Ed., Knowledge Based Learning Systems. New York: Springer-Verlag; 1985.

11. Dietterich, T. G.; London, B.; Clarkson, K.; Dromey, G. "Learning and Inductive Inference," In: P. R. Cohen, E. A. Feigenbaum, Eds., The Handbook of Artificial Intelligence, Volume 3. Los Altos, CA: William Kaufmann, Inc.; 1982:323-511.

12. Michalski, R. S.; Carbonell, J. G.; Mitchell, T. M., Eds. Machine Learning: An Artificial Intelligence Approach. Palo Alto, CA: Tioga Publishing Co.; 1983.

13. Dietterich, T.; Michalski, R. "Learning and Generalization of Characteristic Descriptions: Evaluation Criteria and Comparative Review of Selected Methods," Proc. Sixth Int. Joint Conf. on Artificial Intelligence, 1979, 223-231. 